\title{
EFFECT OF BISPHOSPHONATES ON THE ALVEOLAR BONE OF RATS WITH GLUCOCORTICOIDS INDUCED OSTEOPOROSIS
}

\author{
Hagar S. Abdel Fattah ${ }^{1 *} M S c$, Nawal A. El Masry ${ }^{2} P h D$, Khadiga Y. Kawana ${ }^{2} P h D$, \\ Nesma M. Khalil ${ }^{3} P h D$
}

\begin{abstract}
INTRODUCTION: Bone is a unique tissue that undergoes frequent remodeling. An imbalance between bone formation and resorption results in osteoporosis. Glucocorticoids (GCs) are widely used clinically as anti-inflammatory and immunomodulatory. However, their prolonged use may induce osteoporosis. Currently, evidences revealed that also the alveolar processes are subject to osteoporosis. Bisphosphonates, such as Alendronate, Risedronate and Zoledronate, are recommended by several international guidelines as the first-line therapy for the prevention and treatment of generalized glucocorticoids induced osteoporosis (GIOP).

OBJECTIVES: To evaluate the effect of bisphosphonates (Risedronate) on the alveolar bone of rats with glucocorticoids induced osteoporosis. MATERIALS AND METHODS: Thirty female albino rats were divided into 3 equal groups (10 rats each) as follows: Group I: control group. Group II: glucocorticoids group (Dexamethasone $0.6 \mathrm{mg} / \mathrm{kg}$ twice/week subcutaneously). Group III: Risedronate treated group; rats were given Dexamethasone $(0.6 \mathrm{mg} / \mathrm{kg}$ twice/week subcutaneously) and Risedronate $(1 \mathrm{mg} / \mathrm{kg} /$ day orally). After 12 weeks, rats were euthanized, and the mandibular alveolar bone was evaluated histologically by light microscope and ultra-structurally by scanning electron microscopy and energy dispersive X-ray (EDX) microanalysis.

RESULTS: Group II showed deterioration of the alveolar bone in comparison to the control group I. The alveolar bone structure in Group III rats, treated by bisphosphonates (Risedronate), showed remarkable improvement both histologically and ultrastructurally in comparison to group II. Moreover, EDX analysis revealed a significant decrease of calcium levels in group II in comparison to group I. However, in group III calcium levels were restored to normal levels comparable to the control group I.
\end{abstract}

CONCLUSIONS: Glucocorticoids intake induces loss of the alveolar bone; which, could be counterbalanced by bisphosphonates.

KEYWORDS: Bisphosphonates, glucocorticoids, osteoporosis, alveolar bone.

1. Assistant Lecturer of Oral biology, Faculty of Dentistry, Alexandria University.

2. Professor of Oral Biology, Faculty of Dentistry, Alexandria University.

3. Lecturer of Oral Biology, Faculty of Dentistry, Alexandria University.

*Corresponding author:

E-mail: Hagar.Sherif15@gmail.com

\section{INTRODUCTION}

Bone is a vital dynamic unique tissue that undergoes frequent remodeling with a specific end goal to replace old, micro damaged bone, by new mechanically stronger bone. This procedure is performed by the harmonious balanced activities of clusters of bone resorbing osteoclasts and bone forming osteoblasts. Many bone maladies are due to disorders in bone remodeling; typical examples include postmenopausal and glucocorticoid-induced osteoporosis (1).

Osteoporosis is a chronic systemic skeletal disorder, characterized by bone mass reduction, microarchitectural deterioration, and raised fragility and delicacy. Consequently, the risk of bone fractures is altogether expanded leading to disability, significant morbidity and increased mortality (2-4). Generalized osteoporosis is classified into primary and secondary. Primary osteoporosis can be postmenopausal or senile, while Glucocorticoids (GCs) intake is the most common cause of secondary osteoporosis (5).

Glucocorticoids are widely utilized in clinical practice; they are indicated as therapy for many inflammatory disorders, autoimmune diseases (such as rheumatoid arthritis, systemic lupus erythematosus...), lung disorders (bronchial asthma and chronic obstructive lung diseases), tumors, and organ transplantation $(6,7)$. Despite their unsurpassed anti-inflammatory and immunomodulatory impacts, GCs have substantial adverse outcomes on prolonged usage such as: osteoporosis, glaucoma, diabetes, obesity, myopathy and cardiovascular disease $(8,9)$.

Glucocorticoid-induced osteoporosis (GIOP) is the main cause of secondary osteoporosis before 50 years and the first drug-induced cause of the disease (9). The rate of occurrence of GIOP is approximately $50 \%$ in patients treated for 6 months or longer (10). Fractures are manifested by $30-50 \%$ of patients receiving periodic GC therapy. Fractures frequently occur at sites enriched in cancellous bone, such as the vertebrae and femoral neck (11).

Under physiological concentrations, glucocorticoids are required for normal bone development (11). However, prior and current prolonged exposure to GCs treatment rises the risk of fracture and bone loss (6).

Excess GCs impair bone formation and enhance bone resorption. They affect osteoblasts, by decreasing their differentiation and maturation leading to reduction in their number and function. In addition, they induce the apoptosis bone marrow-derived mesenchymal stem cells (BMSCs), osteoblasts and osteocytes. $(11,12)$.

Indirect effects of glucocorticoids contribute to bone loss as well, such as decreased intestinal calcium absorption, induction of renal calcium excretion, suppression of sex hormones and growth hormones, 
increased parathyroid hormone and decreased calcitonin secretion $(4,13)$.

The Alveolar bone is "a specialized part of the mandibular and maxillary bone that supports the roots of teeth" (14). The quantity and quality of the mandibular bone are fundamental to the planning of prosthetic treatments and implants by dentists (15).

Studies showed that long-term GCs administration induced alveolar bone loss in mice; diminished the cortical bone mineral substance, as well as bone area and strength of rats' mandibles $(16,17)$. Likewise, GIOP can potentiate the destructive impacts of experimental periodontitis on the alveolar bone of rats (18).

Preventive measures are therefore exceedingly recommended to be initiated against corticosteroid-induced osteoporosis as early as possible (13). All international guidelines recommend for GCs-treated patients, to take calcium and vitamin D supplementations; associated with anti-osteoporotic drugs in case of moderate and high fracture risk $(4,19)$. Bisphosphonates, such as Alendronate, Risedronate and Zoledronate, are the most popular antiosteoporotic drugs (6).

Oral bisphosphonates have been recommended as the selected first-line therapy in most clinical situations given their anti-fracture benefit, safety, and low cost; followed by intravenous (IV) bisphosphonates, denosumab, raloxifene, and teriparatide, which have greater harms or burden of treatment (19). Even though, there is still a controversy concerning the benefits of bisphosphonates versus their long term reported complications, including atypical femoral fractures and osteonecrosis of the jaw that result from over suppression of bone turn over (4).

This work was carried out to evaluate the effect of Bisphosphonates (Risedronate) on the alveolar bone of rats with glucocorticoids induced osteoporosis.

\section{MATERIALS AND METHODS}

This study was conducted following the ethical guidelines for conduct of research on experimental animals, by the Faculty of Dentistry, Alexandria University (IRB NO:00010556 - IORG 0008839).

\section{Experimental animals:}

A total of 30 healthy female Albino rats weighting 180-200 grams (approximately 4-5 months of age) were used in this study. Rats were selected from the Institute of Medical Research Alexandria University. They were housed in specially designed wire mesh bottom cages, five animals per cage. The animals were supplied a regular diet and drinking tap water, and maintained under proper conditions of good ventilation during the whole experimental period.

\section{Study design:}

A blinded prospective comparative study was conducted. Rats were randomly divided into 3 equal experimental groups, (10 rats each) as follows:

\section{-Group I: Control group, (NC)}

Rats were given sterile physiologic saline as a vehicle to control the influence of any injection stress on the animals.

\section{-Group II: Glucocorticoids group, (GCs)}

Rats were given Dexamethasone (Amriya for pharmaceutical industries, Alexandria - Egypt) $(0.6 \mathrm{mg} / \mathrm{kg}$ twice/week by subcutaneous injection) for 12 weeks, to induce osteoporosis (20).

\section{-Group III: Glucocorticoids and Risedronate group (GCs+Ris).}

Rats were given Dexamethasone $(0.6 \mathrm{mg} / \mathrm{kg}$ twice/week by subcutaneous injection) and Risedronate (Risaldene, Hikma pharma S.A.E., 6th of October City- Egypt) (1mg/kg/day orally by oral gavage) for 12 weeks $(21,22)$.

Randomization of the animals assigned to different experimental groups was achieved using computergenerated numbers/sampling (23).

\section{Euthanasia of experimental animals:}

After 12 weeks, all rats were sacrificed. The mandible of each rat was dissected out and divided into 2 halves. The right halves were prepared for light microscopic examination, while the left halves were prepared for Scanning Electron Microscopy (SEM) and Energy Dispersive X-ray (EDX) microanalysis.

Tissue preparation for light microscopic examination: $(24,25)$

All specimens were labeled and fixed in 10\% neutral buffered formalin. After fixation, specimens were decalcified, washed, dehydrated in ascending concentrations of ethanol, cleared with xylene, infiltrated and embedded in paraffin wax. Thin sections of $5 \mu \mathrm{m}$ thick were cut mesiodistally using a rotary microtome. Sections were stained with Hematoxylin \& Eosin stains (H\&E) for general histological examination.

Tissue preparation for Scanning Electron Microscopy (SEM): (26)

The left mandibular molar segments with the surrounding alveolar bone were separated and were immediately fixed in $2.5 \%$ glutaraldehyde in phosphate buffer ( $\mathrm{PH} \mathrm{7.2)} \mathrm{and}$ washed twice in the same buffer. The specimens were then dehydrated in graded series of aqueous ethanol solution $50 \%, 70 \%, 90 \%$, and $100 \%$. Then they were air-dried, mounted on aluminum SEM stubs with silver paint and sputter coated with gold using a sputter coater. Specimens were then examined by scanning electron microscope (Joel JSM-5300, Tokyo, Japan) to study the surface characterization of alveolar bone in the different groups.

The surface topography of bone made visible with SEM provides an exciting way to visualize the past metabolic activity of the bone cells over relatively large bone surfaces. Bone surfaces undergoing resorption show scalloped pits, while quiescent bone surfaces appear smooth (27).

Energy Dispersive X-ray (EDX): (28)

Energy Dispersive X-ray is an analytical technique used for the elemental analysis or chemical characterization of a sample. This type of X-ray was used to compare the percentages of calcium and phosphorus in the alveolar bone of all groups.

\section{Statistical analysis:}

Data obtained from EDX microanalysis were collected and analyzed using IBM SPSS software package version 20.0. Descriptive statistics were displayed as means \pm Standard deviations and range (minimum-maximum). $\mathrm{F}$ test (ANOVA) was used to compare the mean values of the normally distributed quantitative variables between the 3 studied groups. While, unpaired $\mathrm{T}$ test was used for pairwise comparisons. Significance of the obtained results was judged at the $5 \%$ level. 


\section{RESULTS}

\section{Histological findings by light microscope:}

Group I (control group): The alveolar bone showed normal architecture revealing regular surface lined by continuous layer of osteoblasts, many deeply stained resting lines indicating active bone formation. Dense bone trabeculae were observed with cellular bone marrow spaces lined by flat endosteal cells. Volkmann canals were noted traversing the alveolar bone and carrying the neurovascular supply from the periodontal ligaments to the alveolar bone (Figure 1).

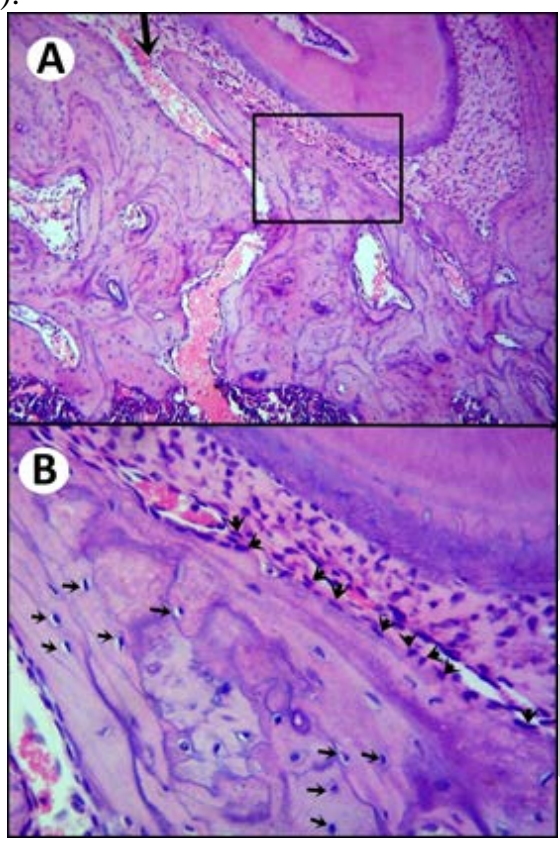

Figure (1): Light micrograph (LM) of the control group I showing the apical region of the alveolar bone surrounding the $1^{\text {st }}$ mandibular molar. A: revealing smooth bone surface, dense bone trabeculae with narrow bone marrow spaces lined by flat endosteal cells. Note resting lines and Volkmann canal (arrow). B: higher magnification of the inset showing regular bone surface lined by continuous layer of osteoblasts (arrow heads) and normal density and distribution of osteocytes (arrows). H\&E, A: X 100, B: X 400

Group II (glucocorticoids group): The alveolar bone showed relatively irregular outline with absence or discontinuity of surface osteoblasts. Bone trabeculae were notably thin surrounding wide bone marrow spaces with fatty tissue infiltration. Moreover, numerous multinucleated osteoclasts in Howship's lacunae were noted, indicating prevailing bone resorption. (Figure 2)

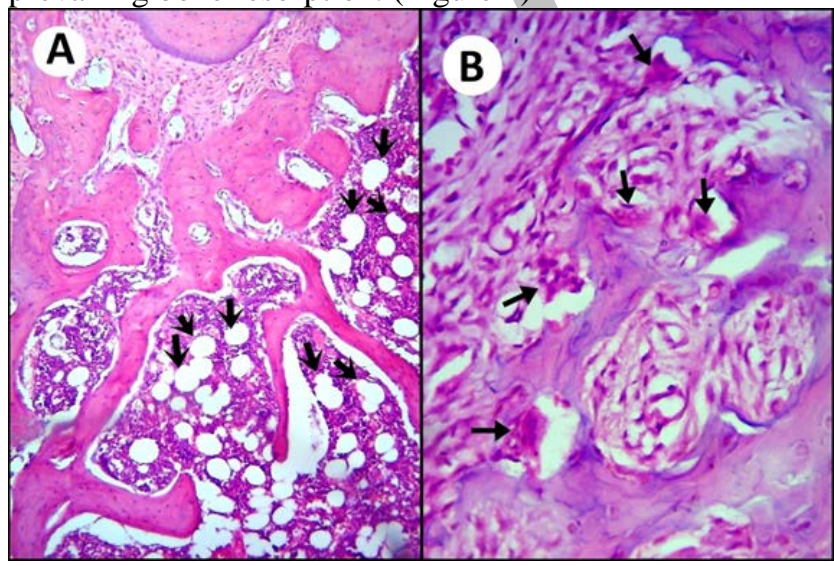

Figure (2): LM of GCs group II showing the apical region of the alveolar bone surrounding the 1st mandibular molar. A: revealing irregular, thin bony trabeculae, surrounding wide bone marrow spaces with fatty infiltration (arrows). B: showing irregular bone surface with multinucleated osteoclasts (arrows). H\&E, A: X100, B: X400

Group III (Bisphosphonates treated group): The alveolar bone normal features were relatively restored. The surface of the alveolar bone was found relatively smooth with discontinuity of surface osteoblasts; darkly stained resting lines were also observed. Dense bone trabeculae were noted with narrow bone marrow spaces. Absence of resorptive concavities as well as osteoclasts was generally noticed; however, enlarged osteocytes lacunae were evident as well as few empty lacunae (Figure 3).

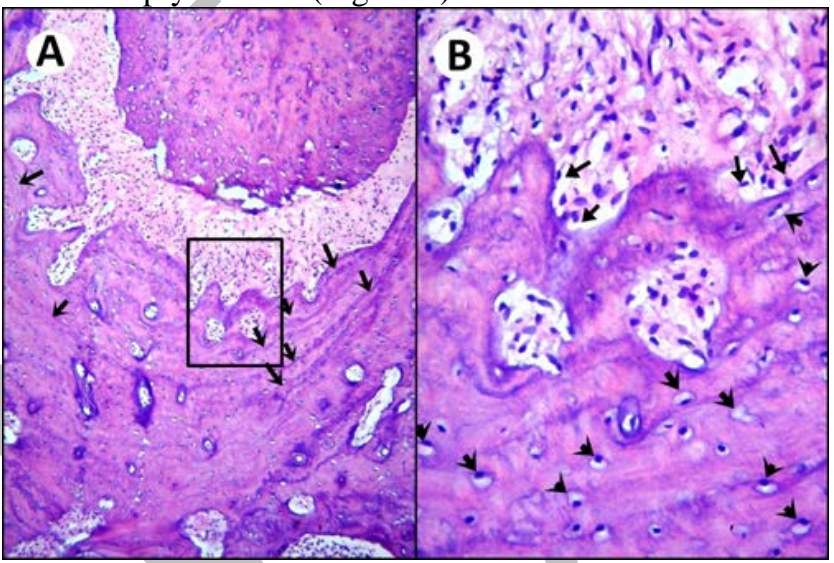

Figure (3): LM of Risedronate treated Group III showing the apical region of the alveolar bone surrounding the 1st mandibular molar. A: revealing relatively smooth and continuous bone surface with many resting lines (arrows). Dense bone trabeculae are noted with narrow bone marrow spaces. B: higher magnification of the inset showing dense bone trabeculae with discontinuity of surface osteoblasts (arrows), and enlarged osteocytes' lacunae (arrow heads). H\&E, A: X100, B: X400

\section{Results of Scanning Electron microscopy: Control group I:}

The surface topography of the buccal cortical plate of alveolar bone showed a generalized smooth, regular, homogenous and uniform surface. It also exhibited nutritive canals having smooth regular borders (Figure 4).

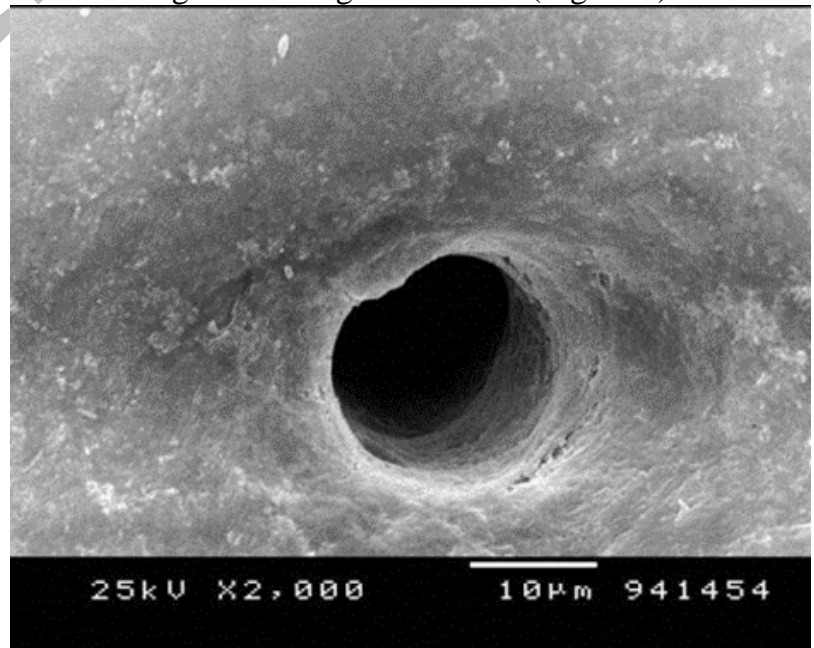

Figure (4): Scanning electron micrograph (SEM) [Control group I] of buccal cortical plate showing generalized smooth, regular and uniform surface topography with a regularly bordered nutritive canal. (X2000) 


\section{Glucocorticoids group II:}

The surface topography of the buccal cortical plate of alveolar bone in this group revealed a generalized pattern of surface roughening and porosity; with prevailing deep irregular pits and wide resorptive craters of different sizes and irregular outline. Pronounced resorption and discontinuation of the bone surface was observed with loss of bone surface architecture. Nutritive canals with osteoporotic changes and wide irregular orifices were also observed (Figure 5).

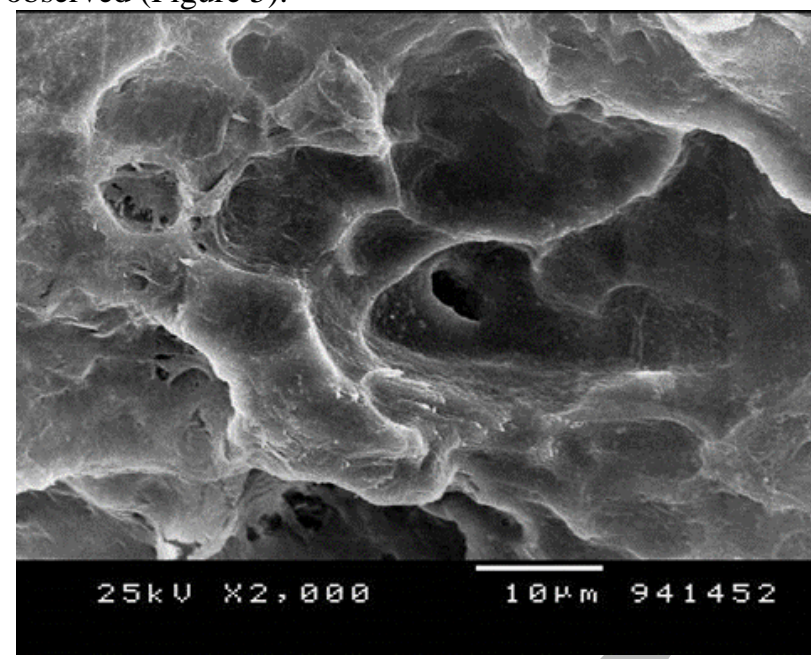

Figure (5): SEM [Glucocorticoids group II] showing irregularity, discontinuation and porosity of bone surface with relatively deep resorption pits and craters. (X2000)

\section{Glucocorticoids and Risedronate group III:}

The surface topography of the buccal cortical plate of alveolar bone in this group revealed alternating areas of smooth surface in association with slightly roughened bone surface with shallow depressions. A decrease in surface roughness and irregularities was noticed. The nutritive canals exhibited a relatively smooth border with slight irregularities interiorly (Figure 6).

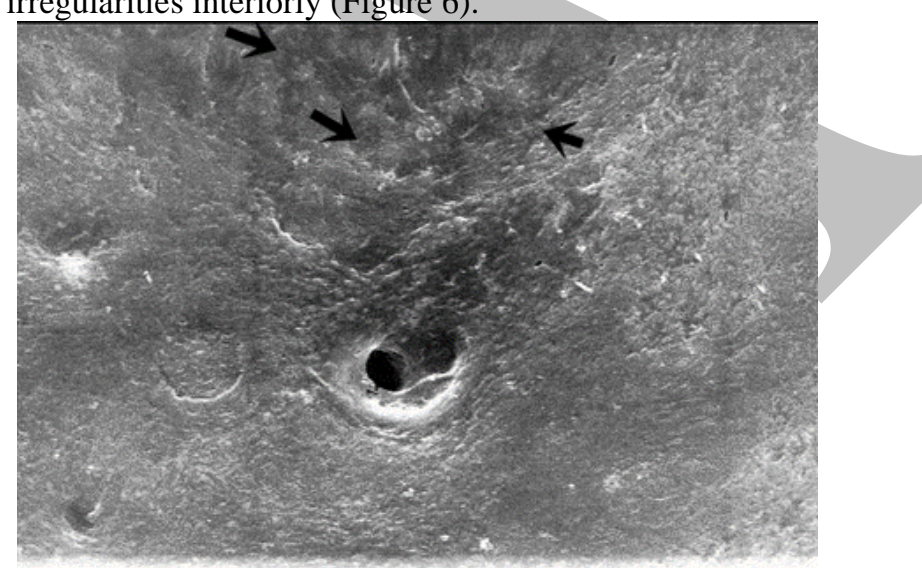

$25 k \cup \times 2,000$

$10 \mu \mathrm{m} 090420$

Figure (6): SEM [Glucocorticoids and Risedronate group III] showing an intact nutritive canal with regular borders surrounded by a relatively smooth and regular bone surface. Note few shallow irregularities (arrows) (X2000)

\section{Results of EDX microanalysis:}

Table 1 shows a comparison among the 3 groups regarding percentages of calcium and phosphorus. Calcium levels in both groups II (GCs) and III (GCs+Ris) were significantly lower than the control group I (P1 <0.05). However, levels of calcium in group III were significantly higher than group II. (P2 <0.05) Phosphorus levels were inversely proportional to calcium levels; moreover, the differences between the 3 groups were statistically significant. (P1 \& p2 $<0.05)$

\section{DISCUSSION}

Osteoporosis is one of the major complications of Glucocorticoids treatment; and represents a global health concern due to the increased propensity to fractures and severe morbidity (4). Bisphosphonates are considered the gold standard in the prevention and treatment of GIOP; Risedronate as well as Alendronate have become the standard care of GIOP. However, the long term use of these drugs may induce osteonecrosis of the jaw specially when associated with the intake of GCs. (6) The current study therefore evaluated the impact of bisphosphonates treatment on the alveolar bone of rats with GCs induced osteoporosis.

The laboratory rat was used as a model in this study because it is convenient, easy and safe to handle, costeffective and is the most frequently used animal model for glucocorticoids induced osteoporosis followed by mice (2931). Dexamethasone was used to induce osteoporosis as it was reported to be the most potent inductor of osteoporosis, among other corticosteroids, in animal models; moreover, it is characterized by its long-term action thus avoiding the need for daily injections $(20,31)$. The dosage and interval used to induce systemic osteoporosis was documented to induced generalized osteoporosis (20,32).

The histological results of the present study revealed noticeable bone resorption and reduction in the thickness of bony trabeculae induced by glucocorticoids administration. Results also showed numerous osteoclasts in Howship's lacunae and absence of continuous surface osteoblasts. Likewise, the scanning electron microscopic examination revealed a porous irregular cortical plate full of deep resorption pits and craters. These results are in agreement with Weinstein et al. 1998 (33) who found that glucocorticoid administration caused reduction in vertebral cancellous bone area and a decline in trabecular width as well as decreased bone formation rate and turnover due to defective osteoblastogenesis determined by ex vivo bone marrow cell cultures. In addition, the mice exhibited an increase in osteoblast and osteocytes apoptosis.

The EDX microanalysis revealed a significant decrease in calcium content in the glucocorticoids group in comparison to the control group. These results are consistent with the study of Kozai et al. 2009 (17) who reported a significant decrease in both the cortical and trabecular bone mineral content and bone area of the mandibular bone on glucocorticoids treatment. Similarly, Hozayen et al. 2016 (34) also reported a significant decrease in bone mineral content of rats' femurs in Dexamethasone group.

Glucocorticoids have been known to enhance bone resorption; by lessening Osteoprotegerin (OPG) expression, increasing receptor of activator of NF-kappa b ligand (RANKL) and macrophage colony stimulating factor (MCSF) expression, and reactive oxygen species (ROS). Furthermore, GCs extend the life span of osteoclasts. In addition, they induce osteoblast and osteocyte apoptosis by increasing pro-apoptotic molecules, reactive oxygen species, and endoplasmic reticulum stress and suppressing 
the Wingless/ Integrated/ $\beta$-catenin (Wnt/ $\beta$-catenin) pathway, a critical pathway for osteoblasts differentiation; thus, impairing osteoblastogenesis (11).

The current study also suggested an increase in fatty tissue infiltrating the bone marrow spaces which was confirmed by Cawthorn et al. 2015 (35) who reported an increase in circulating glucocorticoids causes the expansion of bone marrow adipose tissue. The study of Shata et al.2015 (21) also showed similar results, where they found that the femoral cancellous bone showed severe bone resorption in the form of marked thinning and irregularity of the bone trabeculae. The bone marrow spaces were wider and showed marked increase in the fat area compared with the control group.

In the present study, bisphosphonates showed their capability to preserve the architecture of the alveolar bone, which was manifested by regular bone surface with resting lines indicating bone formation and restoration of normal thickness of bony trabeculae. Moreover, the scanning electron microscopic results showed evident reduction in surface porosity and resorption of the buccal cortical plate, which indicated that bisphosphonates can overcome the destructive effects of GCs. These results were supported by those of Fujita et al.2011 (22), who revealed that Risedronate significantly increased trabecular bone volume, density, and number and reduced trabecular separation. Shata et al. 2015 (21) found matching results in the group treated by Risedronate; the femoral neck showed thicker bone trabeculae and the fat areas in bone marrow spaces were relatively decreased compared with the GCs group. Moreover, numerous dark basophilic cement lines were identified.

However, in the present study few empty lacunae were observed in group III treated by Risedronate. This agrees with Song et al. 2016 (36), who reported the presence of empty lacunae and necrotic bone in mice treated by bisphosphonates following tooth extraction.

Bisphosphonates are thought to work by inhibition of osteoclastic activity. Other proposed mechanisms include alteration of bone turnover and inhibition of angiogenesis (36). Several studies have also suggested that bisphosphonates have a stimulatory effect on osteoblast proliferation and differentiation in vitro. However, there is little evidence to indicate that Risedronate increases bone formation in vivo (22).

In addition, the EDX microanalysis showed that the calcium levels were significantly increased in comparison to the osteoporotic group. These results were supported by those of Fujita et al.2011 (22), who found that Risedronate improved the prednisolone-induced reduction in the mandibular cortical bone surface are as well as mineral content. Misof et al. 2017 (37) also reported an increase in mean calcium content after Risedronate treatment in transiliac biopsies from patients with low bone turnover.

\section{CONCLUSION}

The intake of glucocorticoids induces remarkable loss of the alveolar bone; which, could be counterbalanced by bisphosphonates that prevented cortical as well as trabecular bone loss.

\section{CONFLICT OF INTEREST}

The authors declare that they have no conflicts of interest.

\section{REFERENCES}

1. Jensen PR, Andersen TL, Hauge E-M, Bollerslev J, Delaissé J-M. A joined role of canopy and reversal cells in bone remodeling-lessons from glucocorticoid-induced osteoporosis. Bone. 2015;73:16-23.

2. Oheim R, Schinke T, Amling M, Pogoda P. Can we induce osteoporosis in animals comparable to the human situation? Injury. 2016;47:S3-S9.

3. Zhang Z, Ren H, Shen G, Qiu T, Liang D, Yang Z, et al. Animal models for glucocorticoid-induced postmenopausal osteoporosis: An updated review. Biomed Pharmacother. 2016;84:438-46.

4. Whittier X, Saag KG. Glucocorticoid-induced osteoporosis. Rheum Dis Clin North Am. 2016;42:177-89.

5. Soriano R, Herrera S, Nogués X, Diez-Perez A. Current and future treatments of secondary osteoporosis. Best Pract Res Clin Endocrinol Metab. 2014;28:885-94.

6. Briot K, Roux C. Glucocorticoid-induced osteoporosis. RMD open. 2015;1:e000014.

7. Zhou C, Zhang S, Wang H, Dong L, Zhou G, Song H, et al. Anti-osteoporotic activity of the ethanol extracts of eucommia ulmoides in glucocorticoid-induced osteoporosis male rats through the activation of androgen receptor signaling. Int J Clin Exp Med. 2016;9:2148-56.

8. Seibel MJ, Cooper MS, Zhou H. Glucocorticoid-induced osteoporosis: mechanisms, management, and future perspectives. Lancet Diabetes Endocrinol. 2013;1:59-70.

9. Caplan A, Fett N, Rosenbach M, Werth VP, Micheletti RG. Prevention and management of glucocorticoid-induced side effects: A comprehensive review: A review of glucocorticoid pharmacology and bone health. J Am Acad Dermatol. 2017;76:1-9.

10. Briot K, Cortet B, Roux C, Fardet L, Abitbol V, Bacchetta J, et al. 2014 update of recommendations on the prevention and treatment of glucocorticoid-induced osteoporosis. Joint Bone Spine. 2014;81:493-501.

11. Komori T. Glucocorticoid signaling and bone biology. Horm Metab Res. 2016;48:755-63.

12. Compston J. Management of glucocorticoid-induced osteoporosis. Nat Rev Rheumatol. 2010;6:82-8.

13. Bartl R. Corticosteroid-Induced Osteoporosis. Bone Disorders: Springer; 2017. p. 431-4.

14. Avery JK, Chiego DJ. Essentials of Oral Histology and Embryology: A Clinical Approach: Mosby Elsevier; 2006.

15. Lucinda L, Vieira B, Oliveira T, Sa R, Peters V, Reis J, et al. Evidences of osteoporosis improvement in Wistar rats treated with Ginkgo biloba extract: a histomorphometric study of mandible and femur. Fitoterapia. 2010;81:982-7.

16. Bouvard B, Gallois Y, Legrand E, Audran M, Chappard D. Glucocorticoids reduce alveolar and trabecular bone in mice. Joint Bone Spine. 2013;80:77-81.

17. Kozai Y, Kawamata R, Sakurai T, Kanno M, Kashima I. Influence of prednisolone-induced osteoporosis on bone mass and bone quality of the mandible in rats. Dentomaxillofac Radiol. 2009;38:34-41.

18. Sousa LH, Moura EV, Queiroz AL, Val D, Chaves H, Lisboa $\mathrm{M}$, et al. Effects of glucocorticoid-induced osteoporosis on bone tissue of rats with experimental periodontitis. Arch Oral Biol. 2017;77:55-61.

19. Buckley L, Guyatt G, Fink HA, Cannon M, Grossman J, Hansen KE, et al. 2017 American College of Rheumatology Guideline for the Prevention and Treatment of GlucocorticoidInduced Osteoporosis. Arthritis Care Res (Hoboken). 2017. 
20.Ren H, Liang D, Jiang X, Tang J, Cui J, Wei Q, et al. Variance of spinal osteoporosis induced by dexamethasone and methylprednisolone and its associated mechanism. Steroids. 2015;102:65-75.

21. Shata A, Firgany AE-DL, Abdel-Hamid AA. Ameliorating effect of combination of simvastatin and Risedronate on glucocorticoid induced osteoporosis model in rats. Afr J Pharm Pharmacol. 2015;9:701-10.

22. Fujita Y, Watanabe K, Uchikanbori S, Maki K. Effects of risedronate on cortical and trabecular bone of the mandible in glucocorticoid-treated growing rats. Am J Orthod Dentofacial Orthop. 2011;139:e267-e77.

23. Johnson PD, Besselsen DG. Practical aspects of experimental design in animal research. ILAR journal. 2002;43:202-6.

24. Kumar G. Preparation of Specimens for Histologic Study. In: Kumar G, editor. Orban's Oral Histology \& Embryology. 13th ed. India: Elsevier Health Sciences; 2014. p. 410-6.

25. Feldman AT, Wolfe D. Tissue processing and hematoxylin and eosin staining. In: Day C, editor. Histopathology: Methods and Protocols 1180. New York: Humana Press; 2014. p. 31-43.

26. Murtey MD, Ramasamy P. Sample Preparations for Scanning Electron Microscopy-Life Sciences. Modern Electron Microscopy in Physical and Life Sciences: InTech; 2016.

27. Helen EG, Winston WW. Methods for Transmission and Scanning Electron Microscopy of Bone and Cartilage. In: An YH, Martin KL, editors. Handbook of Histology Methods for Bone and Cartilage: Humana Press; 2003. p. 499-501.

28. Goldstein J, Newbury DE, Echlin P, Joy DC, Romig Jr AD, Lyman CE, et al. Scanning electron microscopy and X-ray microanalysis: a text for biologists, materials scientists, and geologists: Springer Science \& Business Media; 2012.

29. Komori T. Animal models for osteoporosis. Eur J Pharmacol. 2015;759:287-94.

30.Lelovas PP, Xanthos TT, Thoma SE, Lyritis GP, Dontas IA. The laboratory rat as an animal model for osteoporosis research. Comp Med. 2008;58:424-30.

31. Wood CL, Soucek O, Wong SC, Zaman F, Farquharson C, Savendahl L, et al. Animal models to explore the effects of glucocorticoids on skeletal growth and structure. J Endocrinol. 2018;236:R69-R91.

32. Liang D, Ren H, Qiu T, Shen G, Xie B, Wei Q, et al. Extracts from plastrum testudinis reverse glucocorticoid-induced spinal osteoporosis of rats via targeting osteoblastic and osteoclastic markers. Biomed Pharmacother. 2016;82:15160 .

33. Weinstein RS, Jilka RL, Parfitt AM, Manolagas SC. Inhibition of osteoblastogenesis and promotion of apoptosis of osteoblasts and osteocytes by glucocorticoids. Potential mechanisms of their deleterious effects on bone. J Clin Invest. 1998;102:274-82.

34. Hozayen WG, El-Desouky MA, Soliman HA, Ahmed RR, Khaliefa AK. Antiosteoporotic effect of Petroselinum crispum, Ocimum basilicum and Cichorium intybus L. in glucocorticoid-induced osteoporosis in rats. BMC Complement Altern Med. 2016;16:165.

35. Cawthorn WP, Scheller EL, Parlee SD, Pham HA, Learman BS, Redshaw CM, et al. Expansion of bone marrow adipose tissue during caloric restriction is associated with increased circulating glucocorticoids and not with hypoleptinemia. Endocrinology. 2015;157:508-21.
36. Song M, Alshaikh A, Kim T, Kim S, Dang M, Mehrazarin S, et al. Preexisting periapical inflammatory condition exacerbates tooth extraction-induced bisphosphonaterelated osteonecrosis of the jaw lesions in mice. J Endod. 2016;42:1641-6.

37. Misof BM, Blouin S, Lueger S, Paschalis E, Recker R, Phipps R, et al. Baseline mineralizing surface determines the magnitude of the bisphosphonate effect on cortical bone mineralization in postmenopausal osteoporotic patients. J Musculoskelet Neuronal Interact. 2017;17:183.

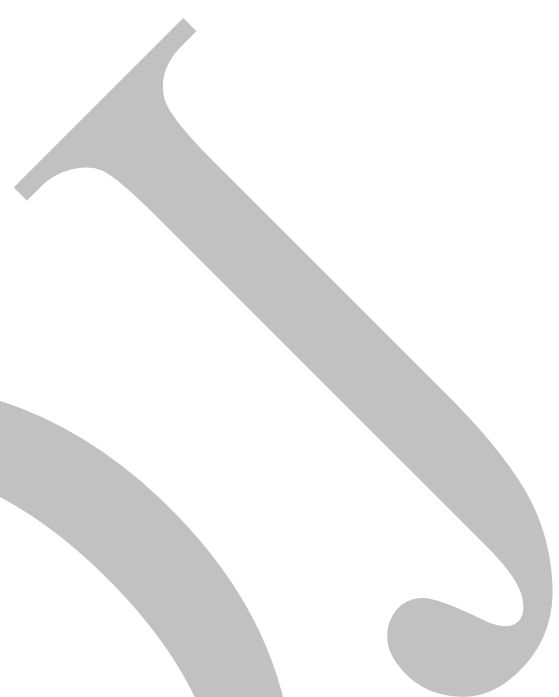

